\title{
Two-photon absorption inside beta- BBO crystal during UV nonlinear optical conversion
}

Sheng Wu, Geoffrey A. Blake, Sunny Sun, Henry Yu

Sheng Wu, Geoffrey A. Blake, Sunny Sun, Henry Yu, "Two-photon absorption inside beta-BBO crystal during UV nonlinear optical conversion," Proc. SPIE 3928, Nonlinear Materials, Devices, and Applications, (23 March 2000); doi: 10.1117/12.379921

Event: Symposium on High-Power Lasers and Applications, 2000, San Jose, CA, United States 


\title{
Two-photon absorption inside $\beta$-BBO crystal during UV nonlinear optical conversion
}

\author{
Sheng $\mathrm{Wu}^{1}$, Geoffrey A. Blake \\ Sunny Sun, Henry Yu ${ }^{2}$ \\ ${ }^{1}$ Division of Geological and Planetary Sciences, California Institute of Technology, CA 91125 \\ ${ }^{2}$ CASIX Inc., 21828 \#D, Lassen St. Chatsworth, CA 91131
}

\begin{abstract}
We measured the two-photon absorption (TPA) cross sections inside $\beta$-BBO crystal during UV harmonic generation. We found that the 2-photon absorption is dominating the absorption effect inside the BBO crystal during UV harmonic generation. Both 2 UV photons and 1 UV photon +1 fundamental photon absorption cross sections are significant. Possible explanations are presented, and compared with other nonlinear optical crystals. Thermal profiles inside the crystals as a result of the strong absorption processes are discussed through a computer program that simulates the heat dissipation process. We conclude that TPA is the significant factor in high power scaling of UV harmonic generation inside nonlinear optical crystals.
\end{abstract}

Key words: two-photon absorption (TPA), BBO, thermal profiles

\section{INTRODUCTION}

High power deep UV sources based on all solid state lasers are desirable in many industrial and scientific fields, e.g., UV lithography, via hole drilling, UV photolysis ${ }^{1}$, LIDAR and many more, for the compact, efficient and cost-effective merits of such systems. With the recent progresses in two fronts --- diode laser pumping and novel nonlinear optical (NLO) crystals (BBO, LBO, CBO, CLBO, etc.), hope is high to achieve high power deep UV through power scaling of such devices. But, as the input power in the visible increases, significant thermal distortion ${ }^{2,3}$ starts to appear --- the conversion efficiency drops, the beam quality degrades, and the stability deteriorates. To overcome such thermal distortions, we have to first understand the thermal sources and profiles behind the nonlinear optical processes.

\section{TPA IN NONLINEAR OPTICAL CRYSTALS}

\subsection{Currently available TPA data in NLO crystals}

Although strong thermal distortions have been observed in BBO, CLBO, not enough quantitative data is available to describe the absorption and thermal profile properties of these two relatively new NLO crystals. There are limited absorption data on these crystals --- particularly TPA cross sections of 2 UV photons, and of 1 UV photon +1 fundamental visible photon at important wavelengths. Here, we measure TPA cross section at two different wavelengths for $\beta$-BBO crystal and demonstrate that TPA cross sections are indeed very important in creating the temperature profile inside the NLO crystals, and they are severely limiting the conversion efficiency at high average power.

Table 1 summarizes the available TPA and temperature bandwidth data of the new NLO crystals (BBO, LBO, CLBO and $\mathrm{CBO}$ ).

Correspondence: sheng@its.caltech.edu 
Table 1. Available nonlinear optical crystals in UV harmonic generation

\begin{tabular}{|c|c|c|c|}
\hline Crystals & Transparency range & TPA cross section data & 266nm Scheme \& Temp. Bandwidth $\left({ }^{\circ} \mathrm{C} / \mathrm{cm}\right)$ \\
\hline$\beta-\mathrm{BBO}$ & $189-3,300 \mathrm{~nm}$ & $0.9 \mathrm{~cm} / \mathrm{GW} \mathrm{w} / 2 \times 266 \mathrm{~nm}^{10}$ & SHG, $4.5^{4}$ \\
\hline CLBO & $180-2,750 \mathrm{~nm}$ & NA & SHG, $6.2^{5}$ \\
\hline $\mathrm{CBO}$ & $170-3,000 \mathrm{~nm}$ & NA & $\mathrm{SFG}, 4.0^{6}$ \\
\hline LBO & $160-2,600 \mathrm{~nm}$ & NA & SFG, $3.8^{7}$ \\
\hline $\mathrm{KD} * \mathrm{P}$ & $<200-2,150 \mathrm{~nm}$ & $0.8 \mathrm{~cm} / \mathrm{GW} \mathrm{w} / 2 \times 266 \mathrm{~nm}$ & SFG, $1.8^{2}$ \\
\hline
\end{tabular}

\subsection{TPA in BBO crystal}

It is already known that the measured linear absorption by BBO crystal at $266 \mathrm{~nm}$ is only about $<0.2 \% / \mathrm{cm}$, but the actual loss of energy during high peak power UV generation is much higher ${ }^{2}$. Obviously, TPA nonlinear process is dominating the process, but it was unclear which TPA process is dominating the absorption. Although there is TPA absorption cross section for BBO, it was only on TPA involving $266 \mathrm{~nm}$. Below is a simple experiment that we conducted. We double a high power dye laser output at $574 \mathrm{~nm}$ (or $287 \mathrm{~nm}$ in the UV). We then measured the power out of the SHG crystal $(8 \times 7 \times 5 \mathrm{~mm}$, type I at $42.8^{\circ}, \mathrm{AR} / \mathrm{AR}$ at $574 \mathrm{~nm}$ and $287 \mathrm{~nm}$ ) at the following 3 conditions:

A. The power is measured right after the first SHG crystal, including both $574 \mathrm{~nm}$ and $287 \mathrm{~nm}$. We observed the change of power as the SHG crystal is tuned for maximum and minimum SHG efficiency.

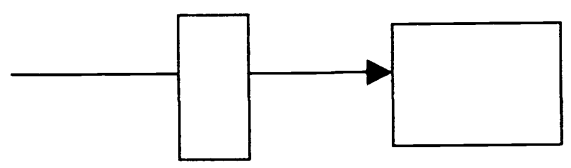

Figure 1. Measurement of difference in power during SHG process.

B. We insert another piece of BBO crystal (cut at another angle, $5 \mathrm{~mm}$ thick, p-coated) after the SHG crystal, and then we measured the total power of $574 \mathrm{~nm}$ and $287 \mathrm{~nm}$ when the SHG crystal is tuned for maximum and minimum SHG efficiency respectively.

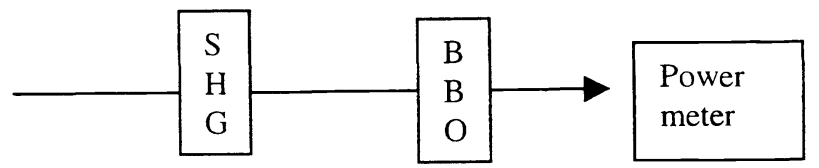

Figure 2. Measurement of difference in power during SHG process and combined 2 photon absorption.

C. We use 2 dichroic mirrors to separate the $287 \mathrm{~nm}$ from the $574 \mathrm{~nm}$, and directed it through the other BBO crystal before the power meter. Therefore only the $287 \mathrm{~nm}$ power is measured when the other BBO crystal is in and out of the beam path. 


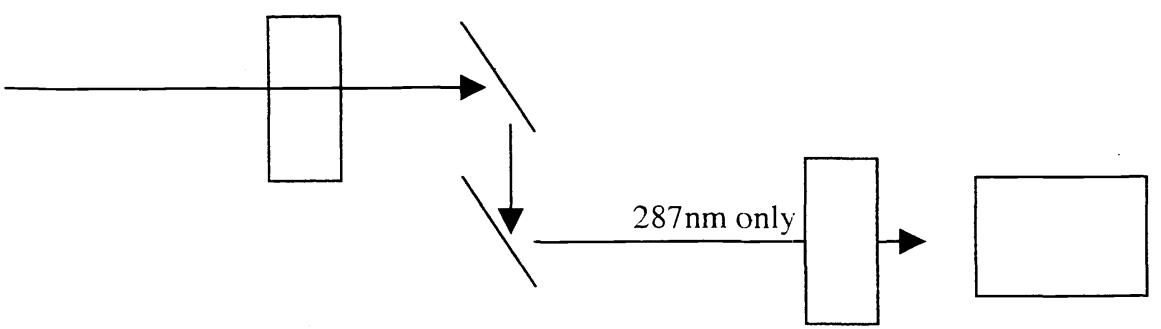

Figure 3. Measurement of the UV power difference after a BBO crystal.

Table 2. TPA measurement data

\begin{tabular}{|c|c|c|c|c|c|c|c|c|c|c|}
\hline & \multicolumn{3}{|c|}{ SHG process, A } & \multicolumn{3}{|c|}{$287+574 \mathrm{~nm}, \mathrm{~B}$} & \multicolumn{4}{|c|}{ Pure $287 \mathrm{~nm}, \mathrm{C}$} \\
\hline $\begin{array}{l}\text { UV Density } \\
(\mathrm{MW} / \mathrm{cm} 2)\end{array}$ & $\begin{array}{c}\text { UV Min. } \\
(\mathrm{mW})\end{array}$ & $\begin{array}{l}\text { UV Max. } \\
(\mathrm{mW})\end{array}$ & $\begin{array}{l}\text { Power } \\
\text { Loss } \%\end{array}$ & $\begin{array}{l}\text { UV Min. } \\
(\mathrm{mW})\end{array}$ & $\begin{array}{l}\text { UV Max. } \\
(\mathrm{mW})\end{array}$ & $\begin{array}{l}\text { Power } \\
\text { Loss\% }\end{array}$ & $\begin{array}{l}\text { W/o xtl } \\
(\mathrm{mW})\end{array}$ & $\begin{array}{l}\text { W/ xtl } \\
(\mathrm{mW})\end{array}$ & $\begin{array}{l}\text { Power } \\
\text { Loss\% }\end{array}$ & $\begin{array}{l}\text { Loss } \\
\text { Inc.\% }\end{array}$ \\
\hline 52.4 & 1945 & 1800 & $7.50 \%$ & 1750 & 1575 & $10.00 \%$ & 655 & 550 & $\begin{array}{r}16.03 \\
\%\end{array}$ & $\begin{array}{r}9.03 \\
\% \\
\end{array}$ \\
\hline 50.8 & 1770 & 1660 & $6.20 \%$ & 1620 & 1449 & $10.60 \%$ & 635 & 535 & $\begin{array}{r}15.70 \\
\%\end{array}$ & $\begin{array}{r}8.80 \\
\% \\
\end{array}$ \\
\hline 47.52 & 1571 & 1466 & $6.70 \%$ & 1426 & 1279 & $10.30 \%$ & 594 & 502 & $\begin{array}{r}15.50 \\
\% \\
\end{array}$ & $\begin{array}{r}8.50 \\
\% \\
\end{array}$ \\
\hline 41.12 & 1299 & 1232 & $5.20 \%$ & 1180 & 1090 & $7.60 \%$ & 514 & 435 & $\begin{array}{r}15.40 \\
\% \\
\end{array}$ & $\begin{array}{r}8.40 \\
\% \\
\end{array}$ \\
\hline 21.2 & 698 & 696 & $0.30 \%$ & 645 & 626 & $2.90 \%$ & 265 & 235 & $\begin{array}{r}11.30 \\
\%\end{array}$ & $\begin{array}{r}4.30 \\
\%\end{array}$ \\
\hline 4.12 & 202 & 201.5 & $0.20 \%$ & 180 & 180 & $0.00 \%$ & 51.5 & 47.9 & $7.00 \%$ & $\begin{array}{r}0.00 \\
\% \\
\end{array}$ \\
\hline
\end{tabular}

We could see from the last column of data that TPA ( 2 photons of $287 \mathrm{~nm}$ ) absorption at $287 \mathrm{~nm}$ is very obvious, because the loss goes up from $\sim 7 \%$ (primarily as a result of Fresnel loss on the surfaces at lower power) to $15.7 \%$ at high power (an increase of $8.8 \%$ ). But in the second and first column, we also observed nonlinear absorption as a result of 1 photon at $287 \mathrm{~nm}$ and 1 photon at $574 \mathrm{~nm}$. It is also very obvious and not negligible compared to the TPA of 2 photons of $287 \mathrm{~nm}$, especially at high input power.

Similar absorption pattern has been observed for BBO crystal at $266 \mathrm{~nm}$, by using the second harmonic output from an Infinity Nd:YAG laser (Coherent Laser, Santa Clara) at $532 \mathrm{~nm}$. The measured TPA cross sections are significant for both $532 \mathrm{~nm}+266 \mathrm{~nm}$ and $2 \times 266 \mathrm{~nm}$ processes. We also did TPA measurement at $355 \mathrm{~nm}$, and only the TPA of $2 \times 355 \mathrm{~nm}$ photons is observed for BBO. The TPA $(2 \times 355 \mathrm{~nm})$ cross section at $355 \mathrm{~nm}$ for BBO is also much smaller (>1 order of magnitude smaller) than that at $266 \mathrm{~nm}$. This is consistent with the Z-scan data ${ }^{10}$.

Obviously, strong TPA is observed when the total energy of 2 UV harmonic photons or 1 UV harmonic photon plus 1 visible photon reaches the cut-off wavelength, which lies around $190 \mathrm{~nm}$, for BBO crystal. This is true at $355 \mathrm{~nm}$, where only small TPA is observed for $2 \times 355 \mathrm{~nm}$ photons, and also obvious at SHG of $287 \mathrm{~nm}$ and $266 \mathrm{~nm}$ when both the $2 \times$ UV harmonic photons or $1 \mathrm{UV}$ harmonic photon +1 visible photon reaches $190 \mathrm{~nm}$.

Based on this observation, we can explain why CLBO outperforms BBO in the FHG and 5HG deep UV harmonics of $\mathrm{Nd}$ :YAG lasers at high power. CLBO has a cut-off wavelength in the deep UV at $180 \mathrm{~nm}$ which is about $10 \mathrm{~nm}$ shorter than BBO. Therefore, at $266 \mathrm{~nm}$, the CLBO's TPA as result of $1266 \mathrm{~nm}$ photon plus $1532 \mathrm{~nm}$ photon (total energy is $177 \mathrm{~nm}$ ) is much weaker than that of BBO. CLBO's TPA as a result of $2 \times 266 \mathrm{~nm}$ could also be somewhat weaker than that of BBO. These assumptions have to be proven, though, by quantitative measurements. According to table 1, CLBO's temperature 
bandwidth is only slightly higher than that of BBO. Considering the crystal lengths used in reference $3 \mathrm{~b}$ and $3 \mathrm{c}$, $\mathrm{BBO}$ 's temperature bandwidth over crystal length ratio $\left(4.5^{\circ} \mathrm{C} / 2 \mathrm{~mm}\right)$ is actually smaller than the ratio for $\mathrm{CLBO}\left(6.2^{\circ} \mathrm{C} / 3.1 \mathrm{~mm}\right)$, while the $3.1 \mathrm{~mm}$ CLBO generates about 2-3 times the power. Therefore, the assumptions above are reasonable.

\section{TEMPERATURE PROFILE AS A RESULT OF STRONG TPA}

Because the TPA is orders stronger than linear absorption, the thermal profile created as a result of TPA will depend strongly on the intensity profile of the UV harmonic, which is resembles a quadratic curve with its maximum at the exit face of the crystal. This dependence will drastically increase the temperature gradient as shown in computer simulation below.

Because the thermal conductivity is crucial in the computer modeling of the power handling capability, it is very important that we have complete and accurate thermal conductivity data on these crystals. Unfortunately, these data are very incomplete and inaccurate. For example, the only available data on thermal conductivity is on $\mathrm{BBO}$ and the two values from two experiments are quite different ${ }^{4,8}$. We use the average of the values given in reference 4 and $8(\sim 1 \mathrm{~W} / \mathrm{m} / \mathrm{K})$, which is inaccurate indeed, but the conclusion that we could draw is qualitative and therefore independent on the values chosen. However, it is desirable to have more accurate and extensive data for all the new NLO crystals. Another important data that we used is the $h$, which is the heat transfer coefficient at the crystal surface. The typical value of $h$ is $22-50 \mathrm{~W} / \mathrm{m} 2 / \mathrm{K}$, with normal air convection ${ }^{11}$.

Figures 4-6 demonstrates that the temperature profiles inside BBO crystal when they absorb the same amount of power. In figure 4 , homogenous heating of the crystal is considere. In figure 5 , inhomogenous heating of the crystal as a result of TPA of 1 UV harmonic photon plus 1 visible fundamental photon is considered. In figure 6, inhomogenous heating of the crystal as a result of TPA of 2 UV harmonic photons are considered. Here, the UV power is set to increase quadratically versus the crystal length, this quadratic increase of UV harmonic power is very close to reality for collimated harmonic generation with low conversion efficiency. It is obvious that TPA absorption gives a temperature gradient at least twice that of linear absorption when the same amount of power is absorbed. Our results also demonstrate that when absorbing the same amount of power, the longer the crystal used the larger the temperature gradient will be --- this explains why much shorter crystal has to be used for higher power scaling. Also, as the crystal lengths increases by a factor of 3 , the temperature gradient goes up to 3 times for homogenous heating of the crystal, and smaller than 3 times for inhomogenous heating of the crystal. Similarly, increasing the absorbed power by a factor of 9 does not increase the gradient by a factor of 9 for inhomogenous heating, while a factor of 9 for homogenous heating. So, it is obvious that compared to homogenous heating of the crystal, inhomogenous heating of the crystal creates a temperature gradient that is harder to eliminate ${ }^{12}$.

The calculation result presented above is also consistent with our assumption that CLBO outperforms BBO in high power UV harmonic generation mainly because of its smaller TPA process. Because in reference $3 \mathrm{~b}$ and $3 \mathrm{c}$, the CLBO crystals used are about 1.5 times of the length of BBO, while CLBO's temperature bandwidth is also 1.5 times of the length of BBO. The rough guess is that if the absorption are the same, then the ratio of temperature gradient to temperature bandwidth is about the same --- meaning that BBO and CLBO should generate the same amount of UV power before saturation occurs. But, in reality CLBO generates over 2 times UV power that of $\mathrm{BBO}$ before saturation occurs. The magnitude of TPA should therefore also be used as a tool to evaluate the quality, suitability of the nonlinear optical crystals for high power UV generation.

The calculation result presented above also explains why $\mathrm{KD} * \mathrm{P}$ is not as good as BBO in high power UV generation ${ }^{2}$. KD*P has about the same TPA as $\mathrm{BBO}^{2}$, but $\mathrm{KD} * \mathrm{P}$ has a temperature bandwidth only half that of $\mathrm{BBO}$. Even worse, to get the same efficiency for collimated beam, $\mathrm{KD} * \mathrm{P}$ has to be at least twice that of $\mathrm{BBO}$. Thus the temperature gradient inside $\mathrm{KD} * \mathrm{P}$ is much larger than BBO. 
The asymmetric temperature gradient created as a result of TPA will create a thermally induced phase mismatch that will degrade the harmonic conversion very quickly. In the adjacent manuscript, we calculate the conversion efficiency when the thermally induced phase mismatch is included in the 3-wave interaction equation.

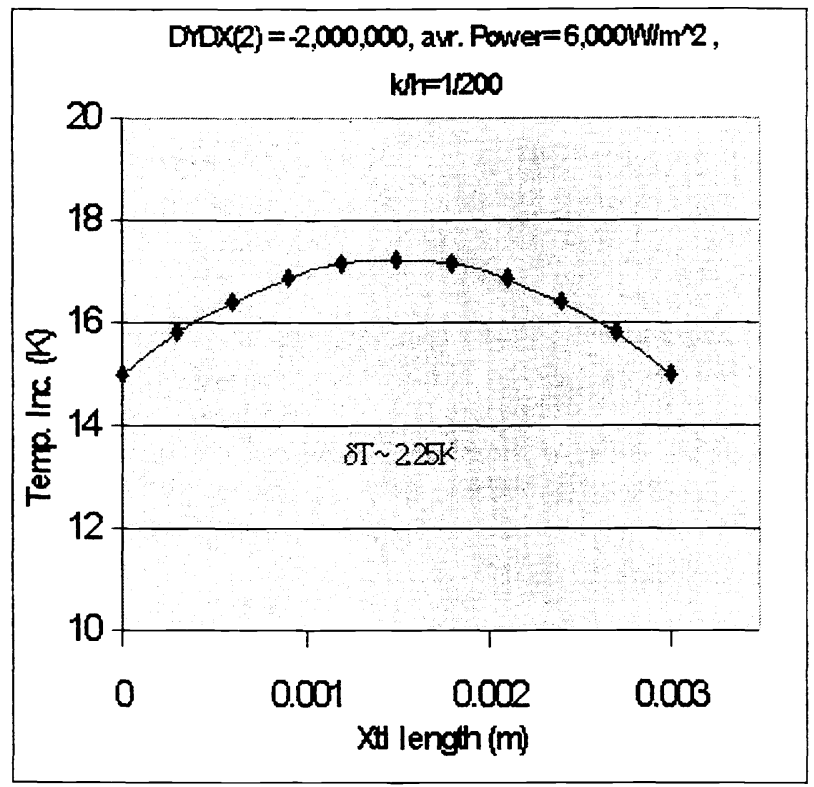

Figure 4a. Linear absorption, $3 \mathrm{~mm}$ xtl, same amount of absorbed power

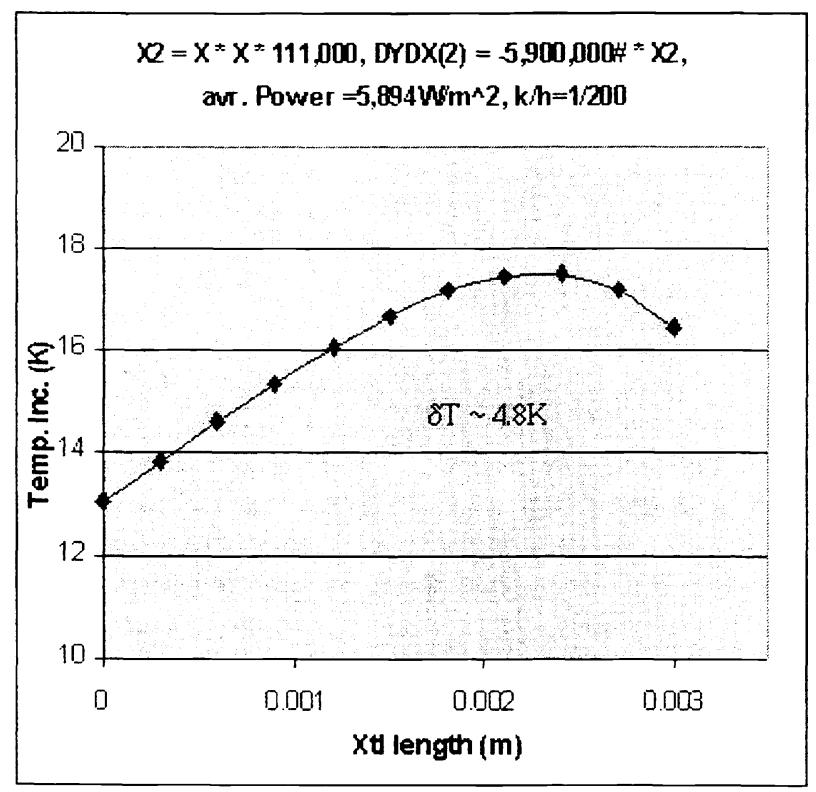

Figure 5a. TPA of $1 \mathrm{UV}+1$ visible, $3 \mathrm{~mm} \mathrm{Xtl}$, same amount of absorbed power

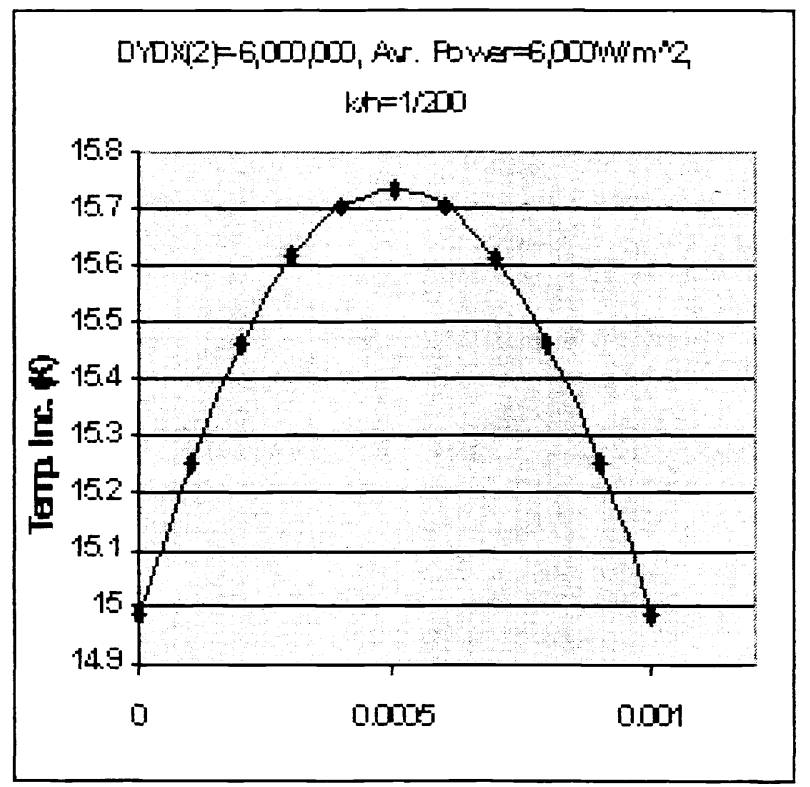

Figure 4b. Linear absorption, $1 \mathrm{~mm} x \mathrm{xtl}$ same amount of absorbed power

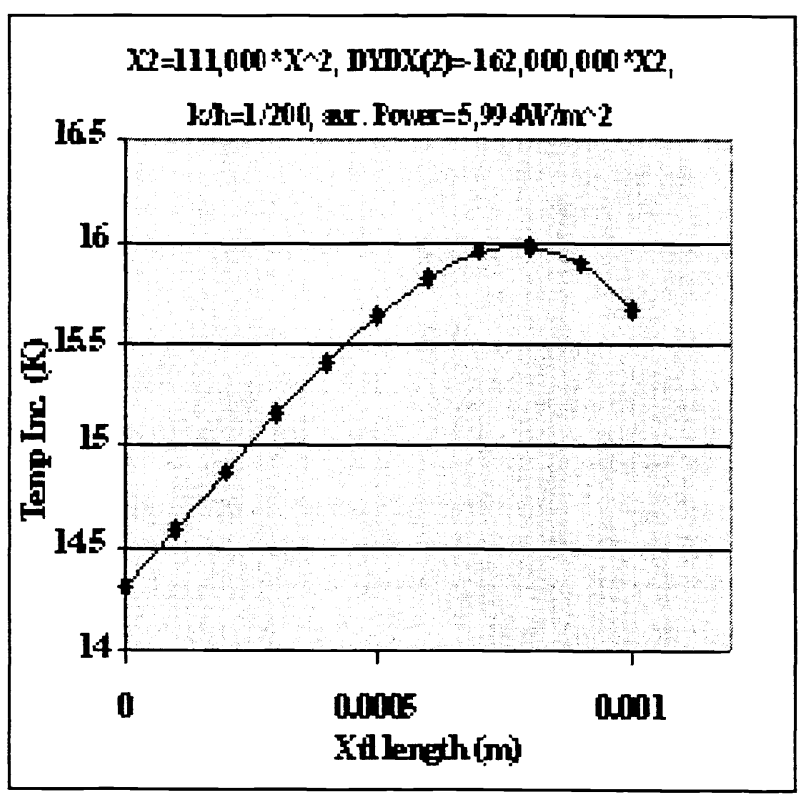

Figure 5b. TPA of $1 \mathrm{UV}+1$ visible, $1 \mathrm{~mm}$ Xtl, same amount of absorbed power 


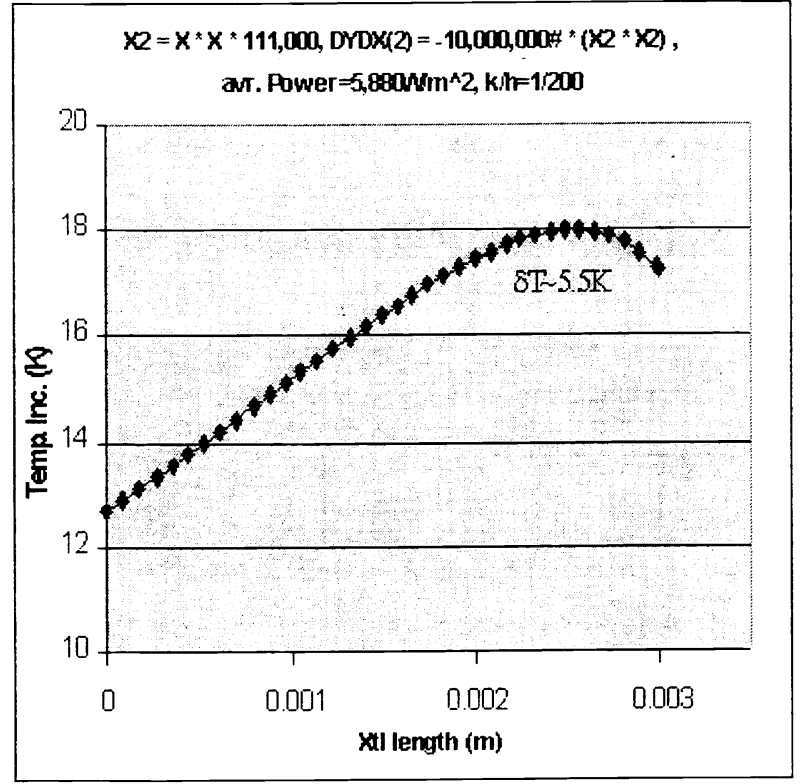

Figure 6a. TPA of $2 x U V$ photons, $3 \mathrm{~mm} x t l$. Same amount of absorbed power

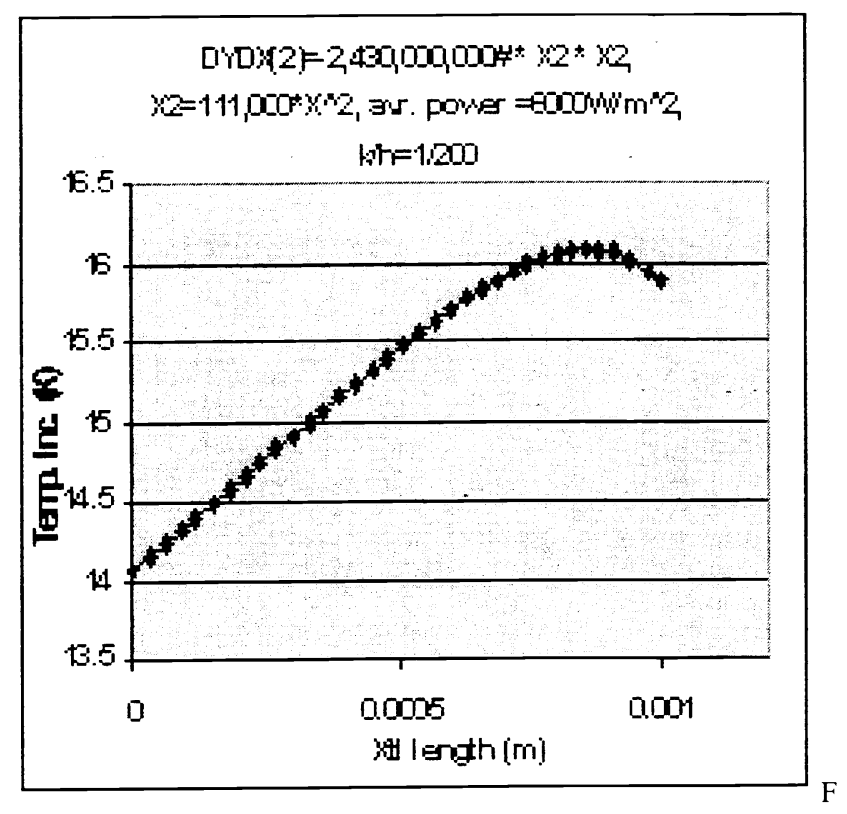

Figure 6b. TPA of $2 x U V$ photons. $1 \mathrm{~mm}$ xtl. Same amount of absorbed power

\section{SUMMARY}

To summarize, we measured the TPA cross sections for BBO in the UV harmonic generation process where both 2 UV photons TPA and $1 \mathrm{UV}$ photon plus 1 visible photon TPA are significant. A computer program is designed to simulate the temperature profile created as a result of such strong TPA processes. The result show that the temperature gradients created as a result of TPA absorption is very asymmetric or inhomogenous, and it is also harder to eliminate than homogenous absorption.

\section{ACKNOWLEDGEMENTS}

The funding from NSF MRI program (grant ATM-9724500, sponsored by the Atmospheric Chemistry program) is gratefully acknowledged.

\section{REFERENCES}

1. Thom Rahn, Hui Zhang, Martin Whalen, \& Geoffrey A. Blake, Geophys. Res. Lett. 25, 4489. (1998)

2. S.C. Matthews and J.S. Sorce, SPIE, Vol. 1220, 137-147 (1990)

3. a. U. Stamm, W.Zschocke, T. Schroder, N. Deutsch, D. Basting, OSA TOPS Vol.10, 7-9, (1997)

b. J.K.Tyminski, C.D. Nabors, G.Frangineas, and D.K.Negus, OSA ASSL, Paper MD2, (1995)

c. Y.K Yap, Y. Mori, S. Haramura, A. Taguchi, T. Sasaki, K.Deki, Y.Ohsako and M. Horiguchi, OSA TOPS Vol.10. 10$13,(1997)$

4. D.Eimerl, L. Davis, S. Velsko, E.K. Graham, A. Zalkin, J. Appl. Phys., Vol. 62, 1968-1983 (1987)

5. N. Umemura et al., OSA TOPS Vol.26 Advanced Solid-State Lasers, 715 (1999).

6. K. Kato "Tunable UV Generation to $0.185 \mu \mathrm{m}$ in CsB3O5", IEEE J. Quantum Elec. Vol. 31, 169-171, 1995

7. K. Kato, IEEE J. Quantum Elec. Vol. 26, 1173-1175, 1990 
8. J.D. Beasley, Appl. Opt. Vol. 33, 1000-1003, 1994

9. Y.K.Yap, K. Deki, N.Kitatochi, Y.Mori, and T. Sasaki, Opt. Lett. Vol.23, 1016-1018, (1998)

10. R. DeSalvo, A.A. Said, D.J. hagan, E. W. Van Stryland, and M. Sheik-Bahae, IEEE J. Quan. Elect. Vol 32, 1324-1333, (1996)

11. P. Kerkoc, S. Horinouchi, K. Sasaki, Y. Nagae, and D. Pugh, J. Opt. Soc. Am. B, 1686-1691, Vol.16, (1999) and private comm. with Dr. Kerkoc

12. David Eimerl, IEEE, J. Quantum Elec. Vol. 23, 575-592, (1987) 